\title{
Thalamic tumours invading the brain stem produce crossed cerebellar diaschisis demonstrated by PET
}

\author{
HIDENAO FUKUYAMA, MASAKUNI KAMEYAMA, KIYOSHI HARADA, \\ NAOKI FUJIMOTO, AKIRA KOBAYASHI, ${ }^{*}$ WARO TAKI,** TSUNEMASA \\ ISHIKAWA,* HAJIME HANDA, * SHUJI TANADA, $\dagger$ KANJI TORIZUKA $\dagger$ \\ From the Department of Neurology, Neurosurgery, ${ }^{*}$ and Nuclear Medicine, $\dagger$ Kyoto University School of \\ Medicine, Kyoto, Japan
}

SUMmaRY Two cases of thalamic tumour, spreading through the midbrain into the pons ventrolaterally, showed suppression of cerebellar blood flow on the opposite side to the tumour, and also showed reduction of blood flow in the pons on the side of the tumour in positron emission tomographic studies. The pathophysiological mechanism of crossed cerebellar diaschisis may be due to functional suppression of the pontine nuclei by destruction of descending fibres from the cerebral cortex.

The purpose of this study was to elucidate the mechanism of crossed cerebellar diaschisis based on positron emission tomographic (PET) findings in three cases of deeply seated brain tumours. It has been reported that crossed cerebellar diaschisis has been observed in cases of frontal or parietal lobe lesions. ${ }^{1-6}$ We have studied two cases of thalamic tumour involving the brain stem in which crossed cerebellar diaschisis was observed, while a third case of thalamic tumour expanding upward toward the lateral ventricle showed no reduction of contralateral cerebellar blood flow. The three cases were examined with oxygen-15 PET; we discuss the patho-physiological mechanism of crossed cerebellar diaschisis, based on the PET findings.

\section{Patients and methods}

Three cases of brain tumour were identified on X-ray CT scans, which were performed a day before PET study. The orbitomeatal line was marked on the CT scan and referenced to the PET study. Eighteen normal volunteers (age $32 \pm 7 \cdot 8$ ), were also examined, and the normal range on cerebellar hemispheric cerebral blood flow (CBF), cerebral metabolic rate of oxygen (CMRO2) and oxygen extraction fraction (OEF) were determined.

Address for reprint requests: Dr H Fukuyama, Department of Neurology, Kyoto University School of Medicine, 54 Shogoin, Sakyo-ku, Kyoto, 606, Japan.

Received 2 July 1985 and in revised form 4 September 1985 . Accepted 10 September 1985
Case 1

A 63-year-old female was admitted because of right hemiparesis, which began with right leg weakness about one week before admission. Neurological examination on admission revealed a mildly decreased level of consciousness, and right hemiplegia including the face. The tongue was deviated to the right, and pupils were anisocoric (right $3 \mathrm{~mm}$, left $2.5 \mathrm{~mm}$ ), but reactive to light. Deep tendon reflexes were exaggerated on the right side, combined with Babinski's sign. Extraocular movements were not limited, and she had no sensory deficits in all modalities. A CT scan showed a thalamic tumour on the left side extending through the midbrain into the pons ventrolaterally (fig l).

\section{Case 2}

A 26-year-old male began to have occipital headache 2 years before admission, and gradually developed right arm weakness and unsteadiness of gait 1 year before admission, with a tendency to fall towards the right side when walking. In June, 1983, hydrocephalus was found by CT and he was treated with bilateral ventricular drainage, but neurological deterioration continued. Dysarthric speech, dysphagia and finally a locked-in syndrome developed. Communication was then possible only by eye movement. A CT scan showed a left sided brain stem tumour extending from the thalamus, locating mainly on the left side of the midbrain and the pons (fig 2).

Case 3

On 7 June, 1984, a 50-year-old female suddenly lost consciousness on a bus, followed by convulsive movements for a few minutes. No paresis was observed after the convulsion. She had also suffered from a similar episode 7 months earlier. Neurological examination revealed no abnormalities, although EEG showed sharp waves bilaterally, most prominent on the right frontal leads. A large tumour in the right 524 


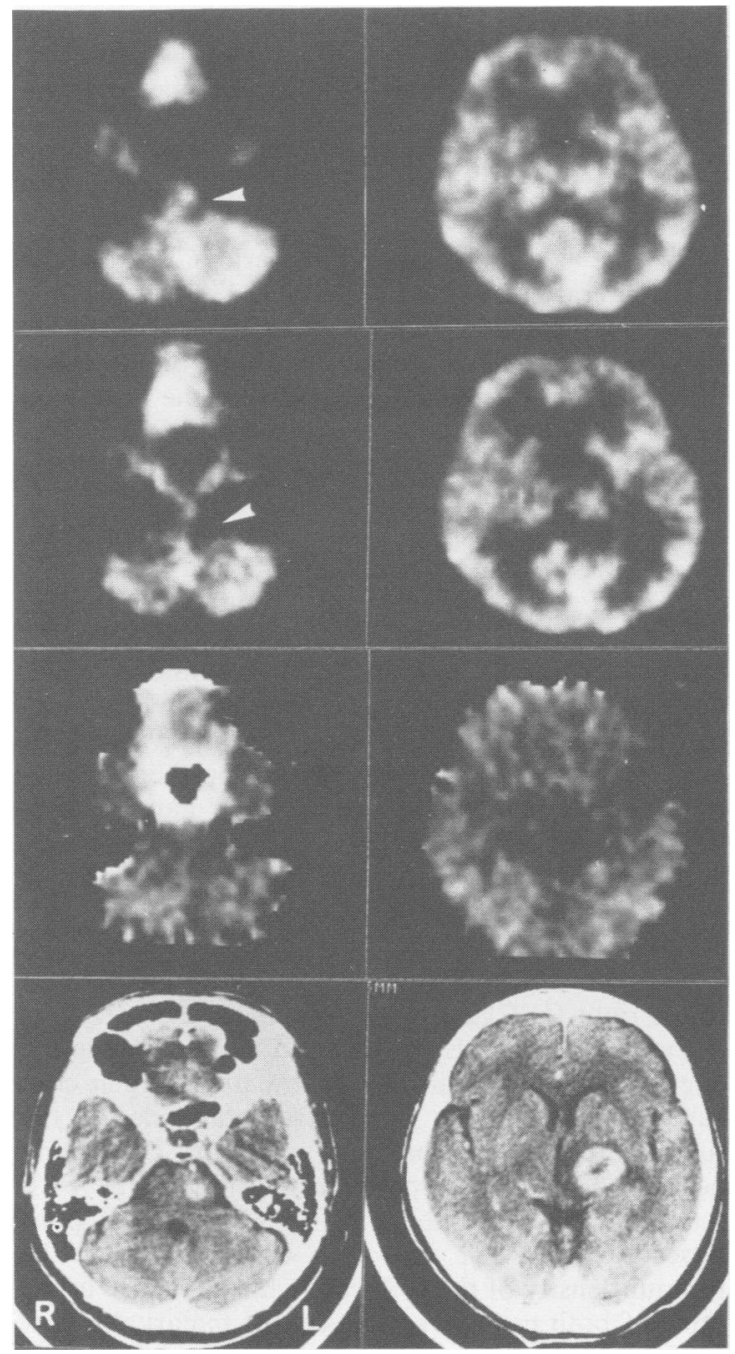

Fig 1 Case 1.63-year-old female, suffered from left thalamic tumour extending downwardly into the pons. Note that the right cerebellum and the left outer part of the pons show reduction of $C B F$ and $C M R O 2$ (arrow). From upper to lower, CBF CMRO2, OEF and CT scan. R in CT scan indicates right side of the brain and Lleft side.

thalamus, extending upward to the corona radiata, was shown by CT (fig 3).

Details of our PET scanner were reported elsewhere. ${ }^{7}$ In brief, it has four rings of 192 bismuth germanate detectors, providing seven slices at one scan process. The best spatial resolution is $7.6 \mathrm{~mm}$ in full width half maximum at the centre of the scan field, and the axial resolution at the centre is $12 \mathrm{~mm}$. Blood sampling was performed two or three times in a scan process from the cannulation of the cubital artery, and oxygen- 15 activity in the whole blood and plasma, as well as arterial blood gases were determined. Prior to the study, a

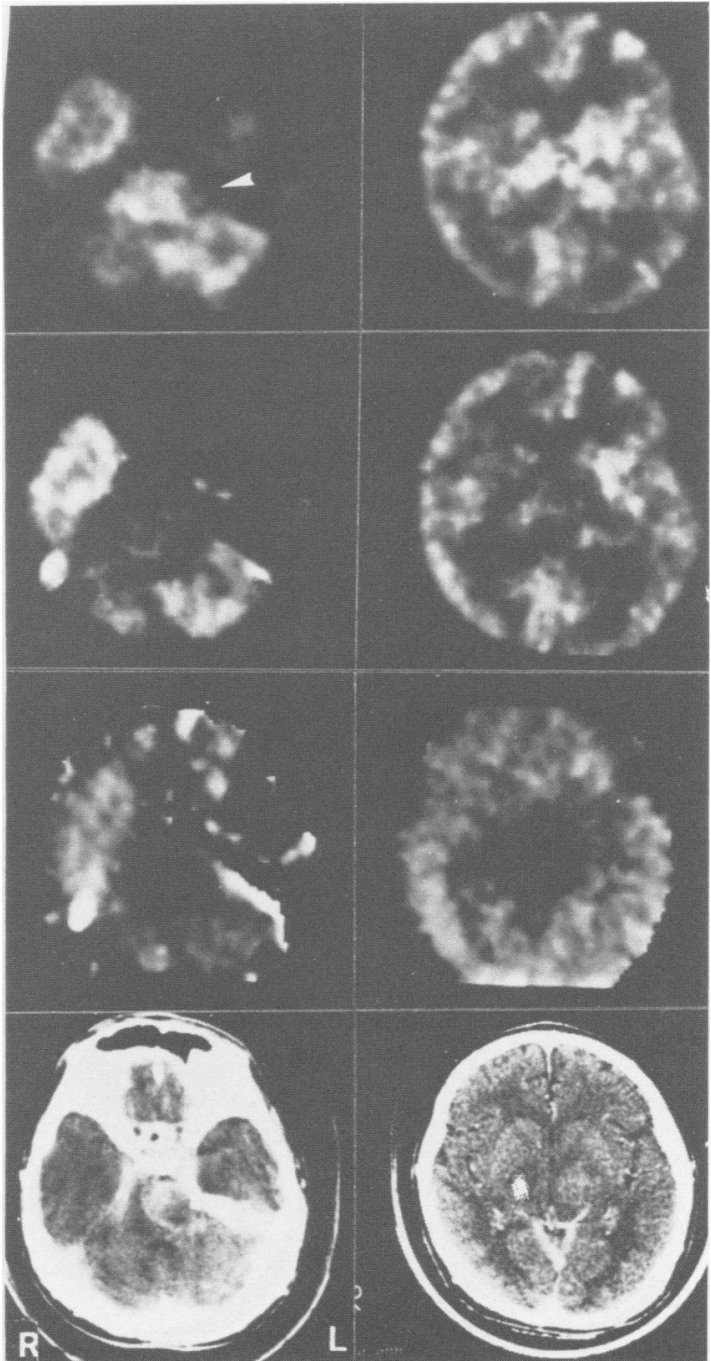

Fig 2 Case 2. 26-year-old male with brain stem tumour involving the thalamus. Note also crossed cerebellar diaschisis and contralateral brain stem suppression of CBF (arrow). Decreased $C B F$ in the left temporal lobe was due to partial volume effect originated from asymmetrical middle cerebral fossa. OEF image of the pons showed remarkable bilateral reduction of $O E F$.

transmission scan using ${ }^{68} \mathrm{Ge}-{ }^{68} \mathrm{Ga}$ was performed for 20 minutes for attenuation correction. Calibration factors between PET scanner and well counter as well as that of cross planes of PET scanner were obtained in every study. Cerebral blood flow was measured by inhaling oxygen- 15 labelled carbon dioxide $10-15 \mathrm{mCi}$ per minute continuously through the mask, and CMRO2 and OEF of the brain by the continuous inhalation of oxygen-15 labelled oxygen $20-30 \mathrm{mCi}$ per minute. Scan data were collected for 5 to 7 minutes. A single breath of oxygen- 15 labelled carbon monoxide $80 \mathrm{mCi}$ 


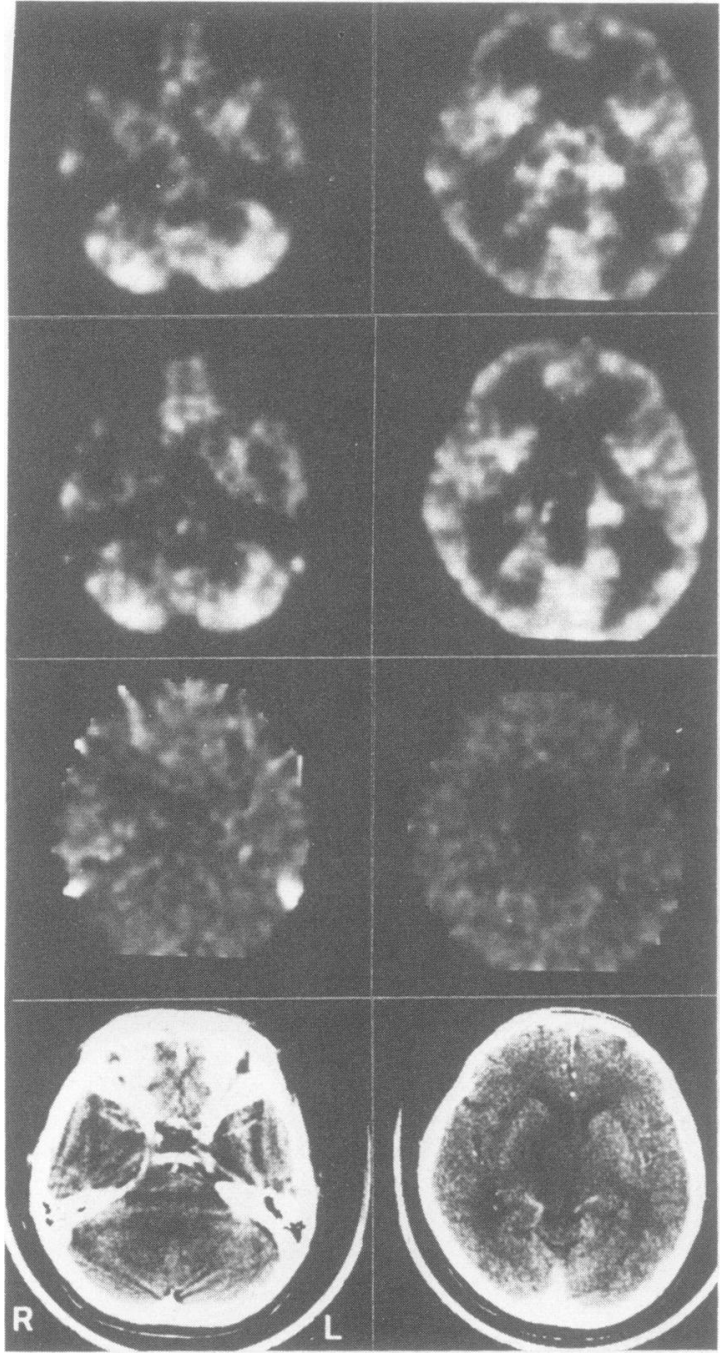

Fig 3 Case 3. 50-year-old female with minimal neurological complications. The tumour in the thalamus without contrast enhancement expands upwardly. Note no crossed cerebellar diaschisis.

was used to measure cerebral blood volume (CBV). Calculation of CBF, CMRO2, and OEF was based on the steady state method, ${ }^{8-10}$ and $\mathrm{CMRO} 2$ and OEF were corrected by CBV value. ${ }^{11} 12$

Image data were processed with a Hitachi image processing computer using system subroutines, in order to reconstruct functional images constituting $128 \times 128$ pixels and calculate mean $\mathrm{CBF}, \mathrm{CMRO} 2$ and $\mathrm{OEF}$ of each cerebellar hemisphere. The region of interest was determined using light pen on both cerebellar hemisphere excluding vermis, so as to cover about $100-150$ pixels.

\section{Results}

The normal value of $\mathrm{CBF}$ in both cerebellar hemispheres from 18 normal volunteers was $39 \cdot 5+9 \cdot 6$ (mean \pm standard deviation) $\mathrm{ml} / 100 \mathrm{ml} / \mathrm{min}$, that of CMRO2 $2.72 \pm 0.49 \mathrm{ml} / 100 \mathrm{ml} / \mathrm{min}$ and that of OEF $0.377 \pm 0.079$

In case 1, cerebellar hemispheric CBF on the contralateral side to the tumour was $28 \cdot 5 \pm 4 \cdot 2$, while that on the ipsilateral side was $38 \cdot 1 \pm 4 \cdot 9$. CMRO2 showed the same tendency; $\mathrm{CMRO} 2$ on the contralateral side was $2.07 \pm 0.38$ and on the ipsilateral side $2 \cdot 59 \pm 0.46$, while ipsilateral OEF was $0.43 \pm 0.057$ and contralateral was $0.40 \pm 0.054$ (fig 1). Case 2 revealed that CBF on the contralateral side to the tumour was $16.0 \pm 3.7$ and on the ipsilateral side $23.2 \pm 2.5, \mathrm{CMRO} 2$ was $1.07 \pm 0.42$ and $1.44 \pm 0.28$ and $\mathrm{OEF}$ was $0.326 \pm 0.10$ and $0.352 \pm 0.06$ respectively (fig 2 ). Those two cases had invasion of the tumour into the midbrain and the pons. In case 2, CBF and CMRO2 decreased remarkably in both cerebellar hemispheres compared with normal values, but more marked reduction was noticed on the side contralateral to the tumour. On the other hand, case 3, which showed no tumour invasion into the brain stem, had a normal range of CBF and CMRO2, that is $47.9 \pm 9.9$ in the right cerebellar hemispheric CBF and $48.0 \pm 13.2$ in the left, $2.85 \pm 0.63$ in the right CMRO2 and 2.79 \pm 0.79 in the left and $0.378 \pm 0.034$ in the right OEF and $0.368 \pm 0.031$ in the left.

Cases 1 and 2 also seemed to show a moderate degree of reduction in blood flow and oxygen consumption within the pons ipsilateral side of the tumour, but these findings were not convincing because of the poor spatial resolution of the PET scanner. The decreased area of CBF in the pons observed by PET, however, coincided with the tumour density of the CT scan, while CMRO2 in the pons of both cases, was reduced to remarkably low levels compared to CBF: laterality was less conspicuous, which could be ascribed to a remarkable reduction of OEF in the pons especially in case 2 . On the other hand, case 3 without crossed cerebellar diaschisis exhibited symmetrical CBF, CMRO2 and OEF patterns of the pons.

Clinical records showed that case 1 and 2 with crossed cerebellar diaschisis showed definite hemiplegia or quadriplegia, in contrast to case 3 who had no remarkable neurological deficits.

\section{Discussion}

Diaschisis was first reported by Von Monakow around the turn of this century. ${ }^{13} \mathrm{He}$ reported that structures remote from a primary lesion in the central nervous system was functionally suppressed by some 
neuronal mechanisms. Crossed cerebellar diaschisis has been defined as cerebellar suppression of the CBF on the side contralateral to a supratentorial lesion. Supratentorial lesions, due to cerebrovascular disorders or cerebral tumours have been investigated by PET scan, as first reported by Baron et al..$^{12} \mathrm{CBF}$, CMRO2 and metabolic rate of glucose of cerebellar hemisphere were reduced on the side contralateral to the supratentorial lesion, mainly located in the frontal or parietal lobes. ${ }^{45}$ These reports favoured the hypothesis that crossed cerebellar diaschisis might be caused by the damage of the descending fibres from the cerebral cortex to the pontine nuclei, but no direct evidence was presented. In another report, deeply seated brain tumours did not show crossed cerebellar diaschisis, but the authors stressed the importance of descending fibre systems from the cerebral cortex. ${ }^{6}$

Afferent fibres to the cerebellum, mainly from the frontal and parietal cortex, descend with pyramidal fibres and end on the ipsilateral pontine nuclei; some part of these fibres descend further onto the inferior olivary nuclei. These nuclei project their fibres transversely to the contralateral cerebellar hemisphere. On the other hand, efferent fibres from the cerebellum originate from the dentate nucleus, crossing the midline in the superior cerebellar peduncle. Some of the fibres terminate in the contralateral red nucleus. The remaining fibres end in the ventrolateral nucleus of the thalamus, which projects diffusely to the cerebral cortex as the thalamic radiations. ${ }^{14}$ On the basis of these fibre connections, we can say that fibres and nuclei in the pons relate only to descending fibres from the cerebral cortex. Therefore, the asymmetrical difference of CBF found in the pons supports the hypothesis that abnormal function of descending tracts produces crossed cerebellar diaschisis.

No asymmetry was found on OEF of the cerebellar hemisphere in any cases, which suggests that CBF and CMRO2 were not decreased by ischaemia produced by the pressure of the tumour. The remarkable bilateral reduction of OEF in the pons in case 2 may suggest either mass effect of the tumour or direct invasion into the contralateral side, which coincided with the clinical finding of a locked-in syndrome. Therefore, in this case, bilateral reduced cerebellar hemispheric CBF and CMRO2 compared with normal values, but more pronounced on the side contralateral to the tumour, were partly due to increased intracranial pressure in the posterior fossa and partly due to bilateral fibre disconnection between cerebellar and cerebral cortex.

In single photon emission CT by the Xe-133 inhalation method, it has been reported that many cases of putaminal haemorrhage show more frequent suppression of contralateral cerebellar blood flow than cases of thalamic haemorrhage. ${ }^{15}$ This result is supportive evidence that crossed cerebellar diaschisis might be caused by the damage of descending fibres from the cerebral cortex to the cerebellum.

Cases 1 and 2 with crossed cerebellar diaschisis presented the definite motor palsy, in contrast to case 3 without crossed cerebellar diaschisis who showed minimal neurological deficits. Considering the intimate relationship of corticopontine fibres with corticospinal and corticobulbar fibres, these clinical findings also suggest that crossed cerebellar diaschisis can result from damage of the descending fibres from the cerebral cortex. This accords with Patronas' clinical observation. ${ }^{6}$

Our results, along with the anatomical considerations, suggest that descending fibres from the cerebral cortex, invaded or destroyed at the brain stem level, suppressed the pontine nuclei which then influenced the function of the contralateral side of the cerebellar hemispheres. Therefore, it can be inferred that the descending fibres play a more important role in producing crossed cerebellar diaschisis than the ascending fibres from the cerebellum.

References

${ }^{1}$ Baron JC, Bousser MG, Comar D, Castaigne P. "Crossed cerebellar diaschisis" in human supratentorial brain infarction. Ann Neurol 1980;8:128.

${ }^{2}$ Baron JC, Bousser MG, Comar D, Castaigne P. "Crossed cerebellar diaschisis" in human supratentorial brain infarction. Trans Am Neurol Assoc 1980;105:459-61.

${ }^{3}$ Lenzi G-L, Frackowiak RSJ, Jones T. Cerebral oxygen metabolism and blood flow in human cerebral ischemic infarction. J Cerebr Blood Flow Metab 1982;2:321-35.

${ }^{4}$ Martin WRW, Raichle ME. Cerebellar blood flow and metabolism in cerebral hemisphere infarction. Ann Neurol 1983;14:168-76.

${ }^{5}$ Kushner M, Alavi A, Reivich M, et al. Contralateral cerebellar hypometabolism following cerebral insult: A positron emission tomographic study. Ann Neurol 1984;15:425-34.

${ }^{6}$ Patronas NJ, Di Chiro G, Smith BH, et al. Depressed cerebellar glucose metabolism in supratentorial tumours. Brain Res 1984;291:93-101.

${ }^{7}$ Senda M, Tamaki N, Yonekura Y, et al. Performance characteristics of Positologica III: a whole body positron emission tomograph. Stroke 1985;9:940-6.

${ }^{8}$ Jones T, Chesler DA, Ter-Pogossian MM. The continuous inhalation of oxygen-15 for assessing regional oxygen extraction in the brain of man. $B r J$ Radiol 1976;49:339-43.

${ }^{9}$ Frackowiak RSJ, Lenzi G-L, Jones T, Heather JD. Quantitative measurement of regional cerebral blood flow and oxygen metabolism in man using ${ }^{15} \mathrm{O}$ and positron emission tomography: Theory, procedure, and normal values. J Comput Assist Tomogr 1980;4:727-36.

${ }^{10}$ Lammertsma AA, Jones T, Frackowiak RSJ, Lenzi G-L. 
A theoretical study of the steady-state model for measuring regional cerebral blood flow and oxygen utilisation using oxygen-15. J Comput Assist Tomogr 1981;5:544-50.

11 Lammertsma AA, Jones T. Correction for the presence of intravascular oxygen-15 in the steady-state technique for measuring regional oxygen extraction ratio in the brain: 1. Description of the method. J Cereb Blood Flow Metab 1983;3:416-24.

${ }^{12}$ Lammertsma AA, Wise RJ, Heather JD, et al. Correction for the presence of intravascular oxygen-15 in the steady-state technique for measuring regional oxygen extraction ratio in the brain: 2 . Results in normal subjects and brain tumour and stroke patients. $J$ Cereb Blood Flow Metab 1983;3:425-31.

13 von Monakow C. Die Lokalisation im Grosshirn und der Abbau der Funktion durch kortikale Herde. Wiesbaden, Bergmann, 1914.

${ }^{14}$ Brodal A. Neurological Anatomy in Relation to Clinical Medicine. 3rd ed. New York: Oxford University Press, 1981:294-393.

${ }^{15}$ Kanaya H, Endo H, Sugiyama T, Kuroda K. "Crossed cerebellar diaschisis" in patients with putaminal hemorrhage. J Cereb Blood Flow Metab 1983;3(suppl 1):S27-8. 